\title{
Risk of aphakic glaucoma after pars plana-lensectomy with and without removal of the peripheral lens capsule
}

\author{
M. Stech ${ }^{1} \cdot$ B. Grundel ${ }^{1} \cdot$ M. Daniel ${ }^{1} \cdot$ D. Böhringer ${ }^{1} \cdot$ L. Joachimsen $^{1} \cdot$ N. Gross ${ }^{1} \cdot$ C. Wolf ${ }^{1} \cdot$ H. Link $^{1} \cdot$ U. Gilles $^{1} \cdot$ \\ W. A. Lagrèze ${ }^{1}$
}

Received: 19 April 2018 / Revised: 30 November 2018 / Accepted: 5 December 2018 / Published online: 10 April 2019

(c) The Royal College of Ophthalmologists 2019

\begin{abstract}
Background The etiology of aphakic glaucoma is unclear. It has been suggested that remaining lens epithelium releases cytokines transducing trabecular meshwork cells. Therefore, we compared two cohorts of children undergoing lensectomy. In cohort 1 , the entire lens including its capsule was removed, in cohort 2 the peripheral lens capsule was left intact, also to facilitate secondary intraocular lens implantation later on.

Methods We included children with uni- or bilateral congenital cataract who underwent lensectomy during the first year of life with subsequent contact lenses fitting. Group 1 comprised 41 eyes, group 2 comprised 33 eyes. In group 1 , the median age at surgery was 4.0 months in unilateral and 3.0 months in bilateral cases 1 , in group $2,8.1$ months and 2.4 months, respectively. The mean follow-up was 12.8 years in group 1 and 9.3 years in group 2 . All cases were analyzed for the prevalence of aphakic glaucoma, for visual acuity and for compliance in visual rehabilitation (contact lens/occlusion therapy).

Results We found no significant difference in glaucoma prevalence between group 1 and group $2(p=0.68)$. The overall glaucoma rate was $26 \%$ after the mean follow-up of 11 years in both groups. In unilateral cases, the median visual acuity was $\log$ MAR 0.7 in both groups. In bilateral cases it was $\log$ MAR 0.4 in group 1 and $\log$ MAR 0.2 in group $2(p=0.05)$.

Conclusions Leaving the peripheral lens capsule intact had no negative effect on the incidence of glaucoma and on resulting visual acuity.
\end{abstract}

\section{Introduction}

Congenital cataract is one of the leading causes of treatable blindness in children worldwide [1]. The incidence of surgery for congenital cataract from three national registries in industrialized countries ranges between 1.5 and 2.0 in 10,000 births [2]. Aphakic glaucoma is a serious complication after congenital cataract surgery. It is well established that its overall risk is about $15 \%$ and that it decreases with age at cataract surgery [3]. An older age at surgery, however, increases the risk of amblyopia. In addition to this,

Theses author contributed equally: Stech M, Grundel B

W. A. Lagrèze

wolf.lagreze@uniklinik-freiburg.de

1 Eye Center, Medical Center, Medical Faculty, University of Freiburg, Freiburg, Germany childhood glaucoma is a risk factor for amblyopia per se [4]. Therefore, the optimal timing of surgery for congenital cataract is still a matter of debate and should be the result of balancing the risks of those two unwanted complications, i.e., glaucoma and amblyopia [5].

The pathophysiology of aphakic glaucoma is yet unclear. Several mechanisms are conceivable: (a) comorbidity of congenital cataract and congenital trabecular meshwork or chamber angle anomaly, (b) early loss of scleral spur tension after lensectomy leading to trabecular meshwork maldevelopment and chamber angle narrowing, (c) chamber angle synechiae induced by cataract surgery, (d) vitreous prolapse into the anterior chamber and (e) a negative effect of remaining and proliferating lens epithelial cells on trabecular meshwork function. The latter hypothesis is supported by experimental studies co-culturing lens epithelial cells and trabecular meshwork cells showing that specific cytokines (TGF-b, IL-4, VEGF) being released from lens epithelial cells induce cytoskeletal changes in the trabecular 
meshwork cells [6]. This concept derived from preclinical data lead us to test this hypothesis clinically by comparing two cohorts of children after lensectomy. Both cohorts were treated in our institution almost identically over almost three decades except for one difference: in the first cohort the entire lens capsule and zonula were removed, whereas in the second group, the peripheral lens capsule remained intact, mainly to facilitate secondary IOL implantation later in life. In addition to this, we performed a multivariate analysis to identify factors influencing visual acuity, also to find out the optimal timing of surgery with regard to visual outcome.

\section{Methods}

\section{Ethics statement}

This investigation and analysis of patient data was approved by the Ethics committee of the Medical Center, University of Freiburg (approval \#237/13) and fully adheres to the tenets of the declaration of Helsinki. Informed parental consent was obtained in each case, if possible from both parents.

\section{Study design and participants}

This study is a monocentric, biphasic, longitudinal cohort study. Children undergoing lensectomy were enrolled consecutively and observed over more than two decades. All children had lens opacities extending over the entire pupil without pharmacologic dilation. No lens opacity allowed preoperative retinoscopy or funduscopy prior to pharmacological dilation. The first group of children was operated on in our institution by a single surgeon between the 1990 and 1999. He had published his technique in detail in 1987 [7]. In brief, the lens is aspirated and fragmented by a vitrectome introduced through a small scleral incision above the pars plicata. A second, similar incision is used for continuous saline infusion. The entire lens capsule is removed, assisted by external scleral indentation. In 2003 we invited all treated children for a follow-up eye examination (group 1). Hence, patients were either 3 years of age or older at first follow-up. Their clinical outcomes were reported in 2004 [8]. Group 2 was operated on between 2000 and 2011 by the senior author leaving the peripheral lens capsule intact, i.e., creating an opening of 6-7 mm diameter in the anterior and posterior lens capsule after complete removal of the lens. During the second half of this period, the 20 gauge vitrectomy system was replaced by a 23 gauge system. Apart from this details, all children were treated in exactly the same way, i.e., fitting of rigid, highly gas permeable contact lenses from one manufacturer within one week after surgery, enforcing compliance as good as possible and adjusting optimal refraction on a continuous basis each month after surgery for the first 3 months, thereafter every third month over the first 2 years after surgery and then every 6 months. In analogy to group 1, all children from group 2 were invited for a follow-up examination in 2014, i.e., again at least 3 years after surgery. Until 2015, most of the patients from both groups presented for further examinations resulting in an effective median follow-up of 12.8 years in group 1 and 9.3 years in group 2 . Overall, the follow-up ranged from 3 to 25 years, the mean follow-up was 11.2 years. Children with comorbidities such as anterior segment dysgenesis and primary congenital glaucoma were excluded. Group 1 contained two eyes of one child, group 2 four eyes of two children with moderate microphthalmia. In group 2, three eyes of unilaterally affected children had mild persistent fetal vasculature.

\section{Clinical assessment at follow-up}

The outcome measures were prevalence of aphakic glaucoma, visual acuity, and compliance in visual rehabilitation (contact lens/occlusion therapy). In detail, we documented the following parameters: Age at surgery [months], compliance $[$ good $=$ no interruption of occlusion/contact lens therapy; bad], refraction [dpt; retinoscopy/autorefraction], visual acuity [logMAR; Lea-test or Snellen chart], stereopsis [Bagolini, prism fusion, random dot], intraocular pressure [mmHg; Goldmann applanation, iCare rebound tonometry], eye length [mm; IOL-Master, ultrasound], corneal curvature [mm; IOL-Master], nystagmus, glaucoma/glaucoma suspects [intraocular pressure repeatedly $>21 \mathrm{mmHg}$ and medical/surgical therapy]. Due to the cohort size and the partially retrospective nature of this study, we pooled glaucoma suspects with repeatedly increased intraocular pressure and glaucoma necessitating pressure lowering therapy.

\section{Statistical analysis}

All descriptive summaries are presented as mean \pm standard deviation. Percentages are calculated to summarize categorical data. The rate of aphakic glaucoma was estimated by means of the Kaplan-Meier method. We used the log-rank test to compare the glaucoma rates between the three equally sized age groups. We eventually fitted a multifactorial linear regression model to identify factors affecting visual outcome. We did not correct for multiple testing due to the retrospective nature of this study. We used the $\mathrm{R}$ platform for all computations (R Core Team (2016). R: A language and environment for statistical computing. $\mathrm{R}$ Foundation for Statistical Computing, Vienna, Austria. URL https://www.R-project.org/). 
Table 1 Baseline characteristics

\begin{tabular}{|c|c|c|c|c|c|c|}
\hline & $\begin{array}{l}\text { Group 1, } \\
\text { unilateral }\end{array}$ & $\begin{array}{l}\text { Group 1, } \\
\text { bilateral }\end{array}$ & $\begin{array}{l}\text { Group 2, } \\
\text { unilateral }\end{array}$ & $\begin{array}{l}\text { Group } 2 \\
\text { bilateral }\end{array}$ & $\begin{array}{l}\text { Both groups, } \\
\text { unilateral }\end{array}$ & $\begin{array}{l}\text { Both groups, } \\
\text { bilateral }\end{array}$ \\
\hline$n$ (eyes) & $11 / 41$ & $30 / 41$ & $15 / 33$ & $18 / 33$ & $26 / 74$ & $48 / 74$ \\
\hline Gender male & $11 / 26(42 \%)$ & & $9 / 25(37.5 \%)$ & & $20 / 51(39.2 \%)$ & \\
\hline $\begin{array}{l}\text { Age at } \\
\text { surgery, months } \\
\text { (median, range) }\end{array}$ & $4.0(0.5-6.5)$ & $\begin{array}{l}3.0(1.0- \\
9.0)\end{array}$ & $\begin{array}{l}8.13(0.97- \\
19.03)\end{array}$ & $\begin{array}{l}2.43(0.63- \\
6.43)\end{array}$ & $\begin{array}{l}4.5(0.5- \\
19.03)\end{array}$ & $\begin{array}{l}2.54(0.63- \\
9.0)\end{array}$ \\
\hline
\end{tabular}

\section{Results}

\section{Baseline characteristics}

The baseline characteristics are summarized in Table 1. In total, 41 eyes were included in group 1 and 33 eyes in group 2. About $40 \%$ of children were male. In group 1 , median age at surgery was 4 months in unilateral and 3 months in bilateral cases. In group 2 , median age at surgery was 8 months in unilateral cases and 2 months in bilateral cases.

\section{Influence of capsule status on the rate of aphakic glaucoma}

The overall incidence of aphakic glaucoma is plotted over the follow-up time span in Fig. 1. Rather soon after surgery a few eyes developed glaucoma (4 of 74 eyes within the first 6 months). For the next 10 years, the event rate of new aphakic glaucoma was relatively low with $75 \%$ of eyes having no glaucoma. Thereafter, a significant number of eyes developed glaucoma resulting in a rate of almost $50 \%$ after 13 years.

In the cox proportional hazards analysis comprising the co-variates age at surgery and status of the lens capsule, the latter did not reveal statistical significance $(p=0.80)$ whereas age at surgery showed a clear trend towards higher age being protective $(p=0.07)$. After the mean follow-up of 11.1 years, the overall rate of glaucoma was $26 \%$. Eyes in group 2 did not develop glaucoma until 2.5 years after surgery. Three children out of group 1 developed glaucoma within a few months after surgery. The rate of aphakic glaucoma is plotted over time of follow-up for groups 1 and 2 in Fig. 2.

\section{Influence of age at surgery on the rate of aphakic glaucoma}

We stratified all eyes from both groups into three equally sized groups (Fig. 3). In those eyes operated on between 2 and 10 weeks of age the rate of glaucoma was $50 \%$ at 13 years. In this subgroup, $15 \%$ developed glaucoma within 6 months after surgery while the rest remained relatively

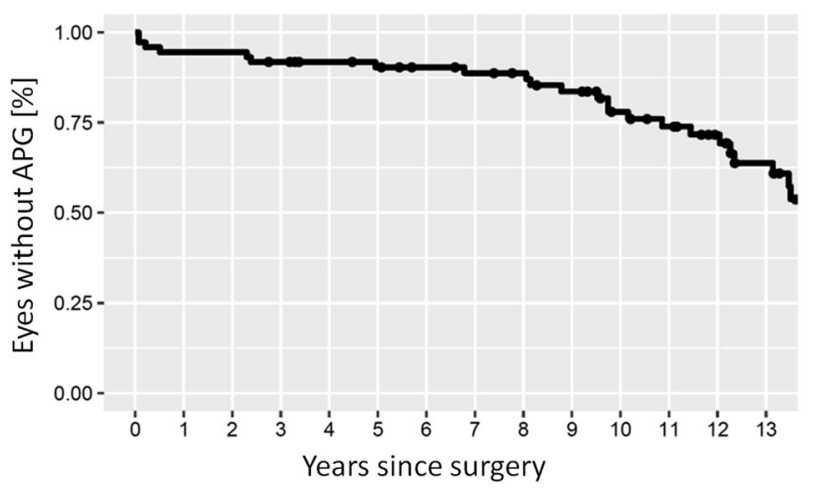

Fig. 1 Kaplan-Meier analysis showing the overall incidence of aphakic glaucoma plotted over follow-up

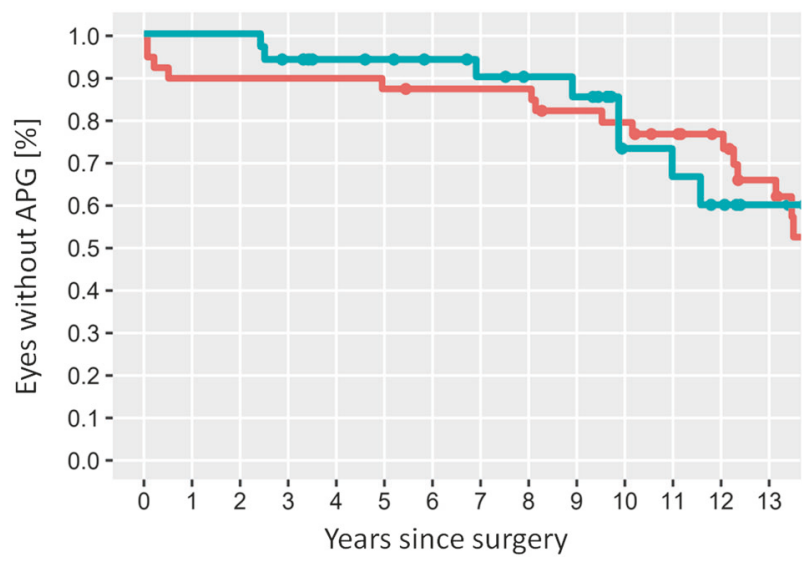

Fig. 2 Kaplan-Meier analysis showing the proportion of eyes after lensectomy without aphakic glaucoma over a follow-up of 13 years. Red line: Eyes with removal of entire lens capsule (group 1). Green line: Eye with remaining peripheral lens capsule (group 2)

stable over the next 10 years. In the ensuing 3 years however, the rate of glaucoma increased markedly. In those eyes operated on between operated between 11 and 20 weeks of age the rate of glaucoma was $27 \%$ at 13 years $(p=0.04)$. The earliest conversion to glaucoma occurred in this group 8 years after surgery. In those eyes operated on after week 21 , the rate of glaucoma was similar to the previous subgroup with a more continuous event rate. We did not observe any retinal detachments during follow-up. 


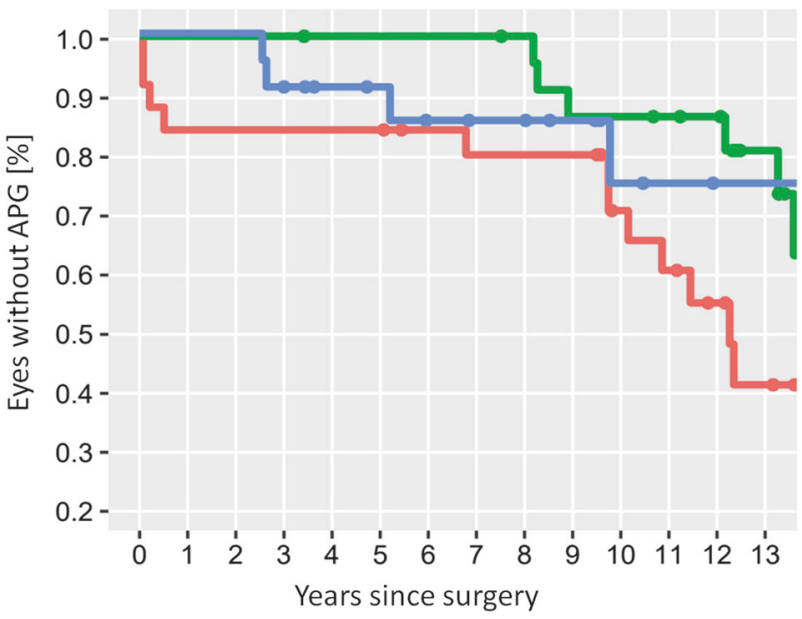

Fig. 3 Kaplan-Meier analysis showing the proportion of eyes after lensectomy without aphakic glaucoma over a follow-up of 13 years. Green line: Eyes operated on at an age of 2-10 weeks. Blue line: Eyes operated on at an age of 11-20 weeks. Red line: Eyes operated on after 20 weeks of age

\section{Visual acuity and amblyopia}

The presence of the lens capsule had a significant effect on visual outcome (linear coefficient $-0.41,95 \% \mathrm{CI}-0.72$ to -0.10 ) while length of follow-up did not influence visual acuity $(-0.02 ; 95 \%$, CI -0.05 to 0.01$)$. Also microphthalmia and persistent fetal vasculature did not show a significant influence on visual acuity or development of glaucoma in our dataset (linear coefficient $0.32 ; 95 \% \mathrm{CI}$ -0.07 to 0.71 respectively $-0.01 ; 95 \%$ CI 0.47 to 0.46 ). Postoperative compliance had a strong influence on visual acuity: bad compliance in either contact lens wear or patching therapy influenced visual acuity with a linear coefficient of 1.68, 95\% CI 1.30-2.07. Compliance was assessed by history.

Figure 4a shows the visual acuity as a function of age at surgery in bilateral cataracts. Focusing at the $95 \%$ confidence interval, excellent visual acuity was achieved when surgery was performed between two and five months of age. When operated at a younger age, the visual outcome was rather variable and ranged between $\log$ MAR 2.7 and logMAR 0.1. Eyes with low visual acuity were those, which developed severe glaucoma. The median visual acuity in bilateral cases was logMAR 0.4 in cohort 1 and $\log$ MAR 0.2 in cohort $2(p=0.05)$, the overall median visual acuity was $\log$ MAR 0.3 .

Children with a unilateral cataract had a more variable outcome as depicted in Fig. 4b. Satisfactory visual acuity was obtained in children undergoing surgery at ages younger than $6-7$ months (visual acuity $<\log$ MAR 1.0 ). In unilateral cases the median visual acuity was $\log$ MAR 0.7 in both cohorts (range logMAR 1.7 to $(-0.1)$ ).

\section{Discussion}

This cohort study was conducted to investigate whether a remaining peripheral lens capsule after lensectomy for congenital cataract, providing a scaffold for proliferating lens epithelial cells, increases the risk for APG, one of the most serious complications after pediatric cataract surgery. The comparison of two groups of children undergoing pars plana lensectomy demonstrated that those with a remaining peripheral lens capsule did not have an increased risk of APG over a rather long mean follow-up period of 11 years with a maximum of 25 years. This set of data derived from 74 eyes after surgery for congenital cataract allows for further analysis for the optimal age of surgery with regard to the risk of glaucoma and amblyopia.

We achieved satisfactory functional outcomes in bilateral cataracts up to an age of 5 months, provided a good compliance in contact lens use and timely adjustment for changes in refraction. On the other side, surgery before 2 months of age was associated with a higher rate of reduced visual acuity at the end of the observational period due to a higher rate of glaucoma. Multifactorial analysis in bilaterally affected children revealed that the main determinant was insufficient compliance in contact lens use and amblyopia treatment.

The rate of APG in our study is in line with previous reports. Trivedi et al. [9] reported at rate of $17 \%$ within 6 years after surgery in 47 aphakic eyes. Of note, all glaucomatous eyes were operated on before the age of 4.5 months. The mean time to diagnosis was 9.8 years. Michaelides et al. [10] reported a rate of glaucoma of $22 \%$ within 5 years. The median age at surgery in eyes with glaucoma was 1.6 months, in those without glaucoma it was 29 months. The authors also stratified their cohort for the status of the posterior capsule. Those with an opening in the posterior capsule had a higher rate of glaucoma indicating that vitreous and soluble factors from the posterior compartment may contribute to trabecular meshwork dysfunction. The authors concluded that lensectomy should not be performed before the age of one month. Lawrence et al. [11] found an overall risk of $11 \%$ within 3 years after surgery at a mean age of 6 months and noted that the risk increases by a factor of 12 when surgery was performed within first month of life. In a large, national population based cohort, Haargaard et al. [3] found a 10-year risk for glaucoma of $32 \%$ when surgery was performed within the first 8 months of life and $4 \%$ when operated on later. Interestingly, in this cohort an increased risk in case of surgery during the first month of life compared to surgery between month 2 and 8 was not observed. The prospective and randomized Infant Aphakia Treatment Study reported a rate of $14 \%$ in the treatment arm receiving no intraocular lens over an observational period of 5 years [12]. Children operated on 


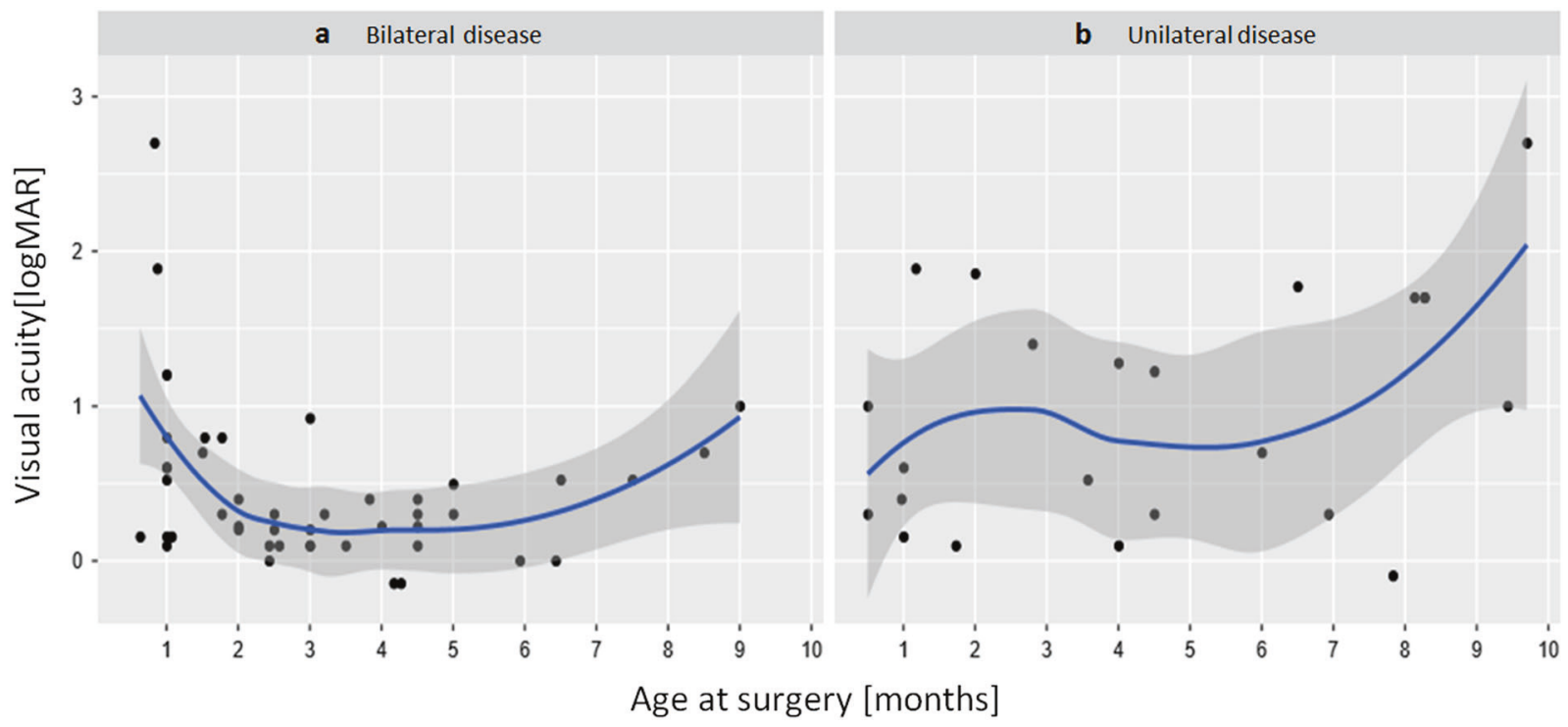

Fig. 4 a, b Visual acuity plotted over age at surgery in children undergoing lensectomy in (a) bilateral and (b) unilateral disease

between 28 and 48 days of age had a threefold increased risk of glaucoma as compared to those operated on after 48 days. The reported glaucoma rates from the aforementioned studies are relatively homogenous and prove the strong influence of the age at surgery, recently confirmed by the very comprehensive meta-analysis by Mataftsi et al. [13] quoting a glaucoma hazard ratio of 2.3 when operated on in the first month of life compared to later surgery. These publications together with our data do not define a clear cutoff time after which lensectomy can be regarded safe concerning the risk of glaucoma. There is rather a continuous decrease in risk with increasing age at surgery, which has to be weighed against the risk of amblyopia and the laterality of the disease.

Because of binocular rivalry, the risk of amblyopia is higher in unilaterally affected children, and it has been shown that good visual acuity can only be achieved when surgery is done not later than six weeks of age [14]. In the Infant Aphakia Treatment Study a weak association of age at surgery and visual acuity was found (cut-off at 48 days of age) [15]. In bilateral disease, several studies have established a well-defined function of visual acuity over time at surgery. Birch et al. [16] described a biphasic function with an initial linear decline of visual acuity at $\geq 5$ weeks of age up to an age of 15 weeks at surgery. Lambert et al. showed that older age at surgery was associated with a decrease in BCVA. However, BCVA of 20/200 or worse only occurred in eyes operated on at ages older than 10 weeks, suggesting that good visual outcomes can be achieved in children undergoing surgery later than 5 to 8 weeks of age [17]. Nystagmus, however, was a significant predictor for a visual acuity $\leq 20 / 100(\operatorname{logMAR} 0.7)$ at an age of 5 years and it was often found in children having undergone surgery at ages over 10 weeks. In our cohort, the visual outcome in bilateral disease has an inversely u-shaped form when plotted over age. Excellent visual acuity could be obtained when surgery was performed within the first 5 months of life. Children undergoing surgery rather early had an increased risk of glaucoma endangering vision over our comparably long follow-up period. This is in agreement with another recent cohort study in 26 bilaterally affected children being operated on in the first 12 months of life, stratified for surgery before or after the age of 10 weeks. At a mean age of 5 years, the rate of amblyopia was similar in both strata. We therefore advocate to re-evaluate current recommendations to treat bilaterally affected children between 6 and 8 weeks of life [5]. Especially in unilateral disease glaucoma and its treatment will affect vision-related quality of life more than amblyopia.

Our cohort study may provide some information on the etiology of aphakic glaucoma. The factor capsule status did not influence the rate of glaucoma. We therefore consider the previously proposed hypothesis unlikely that remaining and proliferating lens epithelia may trigger trabecular meshwork dysfunction. As we also did not observe angle closure, similar to the findings of the Infant Aphakia Treatment Study [12]. We therefore suspect that early surgery interferes negatively with the biomechanics of a still immature anterior chamber angle as recently shown by Chen et al. [18] by ultrasound-biomicroscopy.

Despite a sample size of 74 eyes and a long follow-up of 11 years, conclusions drawn from this investigation are hampered by several factors. We could not randomize both surgical variants in two parallel treatment arms. Therefore it is conceivable that other yet unidentified factors biased the outcome although we could keep all other factors constant over two decades except for the switch from 20 to 23 gauge vitrectomy instruments allowing for a less invasive and 
more minimal surgical manipulation. Secondly, both patients and their examiners were informed about the type of treatment which lies in the retrospective nature of data acquisition, although the analysis was prospectively planned and cases consecutively collected. As in many clinical studies on the long-term outcomes of congenital cataract surgery the precise morphology of the lens opacification was not documented and the rate of uni- versus bilateral cataracts was not ideally balanced in both groups. With regard to the outcome glaucoma, we cannot exclude that some children had coexisting chamber angle anomalies increasing the risk of glaucoma.

In conclusion we believe that we could add some level III-evidence that remnants of the peripheral lens capsule do not increase the risk of APG. An intact peripheral lens capsule rather facilitates secondary implantation of an intraocular lens and avoids the necessity of transscleral suturing. Furthermore, this cohort study provides again arguments against surgery scheduled too early in life carrying the risk of glaucoma induction and re-emphasizes that postoperative compliance is the main determinant for a good functional outcome, provided that glaucoma does not occur. Our set of data also underlines the importance of a lifelong survey of patients after congenital cataract surgery as aphakic glaucoma can occur rather late in life.

\section{Summary}

\section{What was known before}

- Lensectomy early in life contains a high risk of aphakic glaucoma. Its mechanism is yet unknown.

\section{What this study adds}

- This study shows that a remaining peripheral lens capsule providing a scaffold for proliferating epithelial lens cells does not increase the risk of subsequent aphakic glaucoma.

\section{Compliance with ethical standards}

Conflict of interest The authors declare that they have no conflict of interest.

Publisher's note: Springer Nature remains neutral with regard to jurisdictional claims in published maps and institutional affiliations.

\section{References}

1. Sheeladevi S, Lawrenson JG, Fielder AR, Suttle CM. Global prevalence of childhood cataract: a systematic review. Eye Lond Engl. 2016;30:1160-9.

2. Magnusson G, Haargaard B, Basit S, Lundvall A, Nyström A, Rosensvärd A. et al. The Paediatric Cataract Register (PECARE): an overview of operated childhood cataract in Sweden and Denmark. Acta Ophthalmol. (2017). https://doi.org/10.1111/aos.13497

3. Haargaard B, Ritz C, Oudin A, Wohlfahrt J, Thygesen J, Olsen T. et al. Risk of glaucoma after pediatric cataract surgery. Invest Ophthalmol Vis Sci. 2008;49:1791-6.

4. Alsheikheh A, Klink J, Klink T, Steffen H, Grehn F. Long-term results of surgery in childhood glaucoma. Graefes Arch Clin Exp Ophthalmol. 2007;245:195-203.

5. Lambert SR. The timing of surgery for congenital cataracts: Minimizing the risk of glaucoma following cataract surgery while optimizing the visual outcome. J AAPOS. 2016;20:191-2.

6. Michael I, Walton DS, Levenberg S. Infantile aphakic glaucoma: a proposed etiologic role of IL-4 and VEGF. J Aapos. 2011; 48:98-107.

7. Witschel H. Pars plana lentectomy. Klin Mon Für Augenheilkd. 1987;190:406-11.

8. Gessner B, Wiese S, Lagreze WA. Results of pars plana lensectomy for childhood cataract. Ophthalmologe. 2004;101:901-6.

9. Trivedi RH, Wilson ME, Golub RL. Incidence and risk factors for glaucoma after pediatric cataract surgery with and without intraocular lens implantation. J Aapos. 2006;10:117-23.

10. Michaelides M, Bunce C, Adams GG. Glaucoma following congenital cataract surgery-the role of early surgery and posterior capsulotomy. BMC Ophthalmol. 2007;7:13.

11. Lawrence MG, Kramarevsky NY, Christiansen SP, Wright MM, Young TL, Summers CG. et al. Glaucoma following cataract surgery in children: surgically modifiable risk factors. Trans Am Ophthalmol Soc. 2005;103:46-55.

12. Freedman SF, Lynn MJ, Beck AD, Bothun ED, Örge FH, Lambert SR; Infant Aphakia Treatment Study Group. et al. Glaucomarelated adverse events in the first 5 years after unilateral cataract removal in the infant aphakia treatment study. JAMA Ophthalmol. 2015;133:907-14.

13. Mataftsi A, Haidich AB, Kokkali S, Rabiah PK, Birch E, Stager DR Jr. et al. Postoperative glaucoma following infantile cataract surgery: an individual patient data meta-analysis. JAMA Ophthalmol. 2014;132:1059-67.

14. Birch EE, Stager DR. The critical period for surgical treatment of dense congenital unilateral cataract. Invest Ophthalmol Vis Sci. 1996;37:1532-8.

15. Hartmann EE, Lynn MJ, Lambert SR. Baseline characteristics of the infant aphakia treatment study population: predicting recognition acuity at 4.5 years of age. Invest Ophthalmol Vis Sci. 2015;56:388-95.

16. Birch EE, Cheng C, Stager DR, Weakley DR, Stager DR. The critical period for surgical treatment of dense congenital bilateral cataracts. J AAPOS. 2009;13:67-71.

17. Lambert SR, Lynn MJ, Reeves R, Plager DA, Buckley EG, Wilson ME. et al. Is there a latent period for the surgical treatment of children with dense bilateral congenital cataracts? J AAPOS. 2006;10:30-36.

18. Chen D, Gong XH, Xie H, Zhu XN, Li J, Zhao YE. et al. The long-term anterior segment configuration after pediatric cataract surgery and the association with secondary glaucoma. Sci Rep. 2017;7:43015. 